\title{
Structure, rheological, thermal conductive and electrical insulating properties of high-performance hybrid epoxy/nanosilica/AgNWs \\ nanocomposites
}

Chao Chen ${ }^{1,2}$, Hongjian Wang ${ }^{2}$, Yang Xue ${ }^{1}$, Zhigang Xue ${ }^{1, *}$, Hongyuan $\mathrm{Liu}^{2}$, Xiaolin Xie ${ }^{1, *}$, Yiu-Wing $\mathrm{Mai}^{2}$

${ }^{1}$ Key Laboratory for Material Chemistry of Energy Conversion and Storage, Ministry of Education, School of Chemistry and Chemical Engineering, Huazhong University of Science and Technology, Wuhan 430074, China

${ }^{2}$ Centre for Advanced Materials Technology (CAMT), School of Aerospace, Mechanical and Mechatronic Engineering J07, The University of Sydney, Sydney, NSW 2006, Australia

Corresponding authors: Tel: +8627 87793241, Fax: +862787543632

E-mail addresses: zgxue@mail.hust.edu.cn (Z.Xue); xlxie@mail.hust.edu.cn (X. Xie)

Abstract: A facile and effective approach by incorporating silica nanoparticles (SNPs) to fabricate high performance epoxy-based electronic packaging materials which are both thermally conductive and electrically insulating was presented. Because of the strong interaction between SNPs and silver nanowires (AgNWs), uniformly dispersed SNPsmodified epoxy was employed to promote the dispersion of AgNWs in epoxy matrix. Further, the enhanced modulus of epoxy matrix by the incorporation of SNPs effectively alleviates the modulus mismatch between stiff AgNWs and epoxy matrix. Compared with epoxy/AgNWs composites without SNPs, the resulting hybrid materials, that is, epoxy/SNP/AgNWs, showed distinct improvements in thermal conductivity without degrading their mechanical properties. Also, the SNPs were absorbed onto the surface of AgNWs forming an electrical insulation layer to disrupt the electron flows between adjacent AgNWs, hence retaining the electrical insulation of epoxy matrix. Finally, this new fabrication method is easily scalable owing to its simple procedure and use of commercial well-dispersed SNPs-modified epoxies.

Keywords A. Functional composites; B. Nano composites; C. Thermal properties, D. Mechanical properties; E. Rheology 


\section{Introduction}

Miniaturization and three-dimensional integration of modern electronic devices make heat dissipation a significant issue to ensure their high performance and structural reliability in the electronics industry [1-3]. Therefore, there is an urgent need to design epoxy-based underfill materials (EUMs) with high thermal conductivity to dissipate the thermal energy generated by microelectronic chips and cool the electronic devices. However, the poor thermal conductivity of epoxy and the complex packaging process have limited their progress [4]. So far, EUMs still face challenges to meet the stringent requirements for the next-generation electronics in terms of high thermal conductivity $(\kappa \geq 1 \mathrm{~W} / \mathrm{mK})$, high glass transition temperature $\left(T_{g}>125\right.$ $\left.{ }^{\circ} \mathrm{C}\right)$, high electrical insulation and low viscosity $\left(\lambda<20 \mathrm{~Pa} \mathrm{~s}\right.$ at $\left.25^{\circ} \mathrm{C}\right)[5]$.

There have been many attempts to achieve these goals by adding high thermal conductive ceramic fillers into epoxy matrices, e.g., aluminum nitride (AlN) [6], aluminum oxide $\left(\mathrm{Al}_{2} \mathrm{O}_{3}\right)$ [7] and silicon carbide [8,9]. However, high ceramic filler loading is required to achieve the percolation threshold to reach high thermal conductivity. But in doing so, this increases the viscosity of the composite leading to degradations of processability and mechanical properties. One-dimensional nanostructured carbon nanotubes (CNTs) have attracted special interests due to their remarkably high thermal conductivity $(3000 \mathrm{~W} / \mathrm{mK})$ and high aspect ratio that enables easy formation of a heat conducting network with low filler loading [10-12]. However, test results showed that the enhancement of thermal conductivity obtained for the polymer/CNTs composites was lower than the expected value due to intrinsic defects, agglomerated CNTs, and high contact resistance at polymer/CNT and CNT/CNT interfaces [13-16]. Besides, many other kinds of high intrinsic thermal conductivity fillers including one-dimensional BN nano- 
tubes and two-dimensional graphenes have been used to improve the thermal conductivity properties of epoxy composites [17-19]. Nonetheless, the ultrahigh specific surface energy of these nanotubes and nanosheets restricts their homogeneous dispersion in polymer matrices, yielding uncertainties in their thermal conductivity. Silver nanowires (AgNWs), despite their very desirable thermal conductivity as fillers, also have to address the same issues. First, filler agglomerations due to unfavorable interactions between hydrophilic $\mathrm{AgNW}$ s and hydrophobic epoxy matrix and the depletion-induced interaction among AgNWs impart negative influences on the high aspect ratio of the nanowires and the percolation threshold of the composites [20, 21]. Second, the high electrical conductivity of AgNWs limits their application in electronic packaging.

In recent studies $[10,22,23]$, we demonstrated that silica-coated multi-walled carbon nanotubes (MWCNT@SiO 2$)$ and silica-coated silver nanowires $\left(\mathrm{AgNWs} @ \mathrm{SiO}_{2}\right)$ could improve the thermal conductivity and retain electrical insulation of epoxy composites. We further showed that the less stiff silica intermediate nanolayer on CNTs and AgNWs not only alleviated the mismatch between the filler and the matrix, but also enhanced their interfacial interaction. However, the complicated multi-step filler insulation treatment is unsuitable for large-scale applications. Since interaction exists between the SNPs and polyvinyl pyrrolidone (PVP) coated on AgNWs, the SNPs distributed homogeneously in epoxy act as an "inorganic dispersion agent" to improve the dispersion of AgNWs in epoxy. In this work, a facile and effective strategy is proposed for improving the dispersion of AgNWs via SNPs in epoxy to increase the thermal conductivity and retain the mechanical properties of epoxy composites. 


\section{Experimental Work}

\subsection{Materials}

Ethylene glycol (EG, 99.0\%), silver nitrate $\left(\mathrm{AgNO}_{3}, 99.5 \%\right)$ and poly(vinylpyrrolidone) (PVP, $M_{\mathrm{w}} \approx 40000$, powder) were obtained from Sinopharm Chemical Reagent Co., Ltd. (SCRC, China). The bisphenol-F epoxy resin with an epoxide equivalent weight of 170 (YDF-170) was purchased from Kukdo Chemical Co. Ltd., China and the hardener used was a 2-ethyl-4-methylimidazole (EMI-2,4, Beijing Chemical Reagent Co., China). Nanopox F400 containing $40 \mathrm{wt} . \%$ silica nanoparticles (SNPs) with an average diameter of $\sim 20 \mathrm{~nm}$ were supplied by Nanoresins AG, Germany. SNPs with a narrow range of size distribution were synthesized from aqueous sodium silicate solutions by sol-gel method, after undergoing a process of surface modification with organo-silane and matrix exchange to produce an agglomerate-free epoxy/nanosilica master batch [24]. Polyvinylidene fluoride (PVDF) microfiltration membrane with $3 \mu \mathrm{m}$ pores was purchased from Shanghai Xingya Purification Material Factory, China. All other chemical regents were used as- received.

\subsection{Preparation of hybrid epoxy/silica/AgNWs nanocomposites}

Diluted epoxy/nanosilica composite resins with 5, 10 and 15 wt.\% SNPs (designated as F050, F100 and F150) were prepared by mixing Nanopox F400 and YDF-170 with a THINKY centrifugal mixer (Are-310, Japan) at $1600 \mathrm{rpm}$ for 2-minute mixing and at 2000 rpm for 1-minute de-foaming. These procedures were repeated twice to obtain the desired loading of epoxy/SiO 2 nanocomposites with contents of 5, 10 and 15 wt.\%, respectively. From Fig. S1, the homogeneous dispersion of spherical SNPs with very narrow particle size distribution remains unchanged even after dilution. 
AgNWs suspension (see Supporting Information) and a desired amount of epoxy/nanosilica composites (F050, F100 and F150) were mixed followed by ultra-sonication for $2 \mathrm{~h}$ at $50{ }^{\circ} \mathrm{C}$ and then heated to $90{ }^{\circ} \mathrm{C}$ with magnetic stirring for $2 \mathrm{~h}$. The resulting mixture was degassed at $60{ }^{\circ} \mathrm{C}$ to eliminate trapped air bubbles and residual ethanol. The blend was then mixed with 6 wt.\% of EMI-2,4 relative to epoxy, and the mixture was cast in steel moulds and cured at 60 ${ }^{\circ} \mathrm{C}$ for $2 \mathrm{~h}$, followed by post-cure at $150{ }^{\circ} \mathrm{C}$ for $8 \mathrm{~h}$ to complete the curing cycle. Finally, disc -like samples with diameter $100 \mathrm{~mm}$ and AgNWs loadings of 0.5, 1, 2 and 4 vol.\% were prepared for three hybrid epoxy/silica/AgNWs composites. For comparison, epoxy/nanosilica composites and epoxy/AgNWs composites with the same filler loadings were fabricated as controls following the same procedure described above.

\section{Results and discussion}

\subsection{Interaction between AgNWs and SNPs}

It can be seen from the TEM images of the AgNWs (Fig. S2a) that a 1 2 nm thin PVP layer covers the metallic surface; and the TGA curve (Fig. S2b) further shows the amount of PVP adsorbed on the surface is $\sim 2$ wt. $\%$. Because of the O-Ag coordination bonds, a little amount of chemisorbed and physisorbed PVP is coated on the surface of AgNWs [25-27]. Hence, the negatively charged oxygen atoms of the inner amide in PVP can easily form intra-molecular hydrogen bonds with the hydroxyl groups of the SNPs [28,29] (see Fig. S3). Moreover, Monte Carlo simulations have also confirmed that the effects of the attractive van der Waals forces at short lengths exist between AgNWs and SNPs have greatly weakened the depletion-induced interaction among AgNWs, which is responsible for the better dispersion of AgNWs [20]. 
After the AgNWs suspensions were blended with F050, F100 and F150 composites, the mixtures were washed with ethanol and filtered using a $3 \mu \mathrm{m}$ PVDF microfiltration membrane for 3 cycles to remove epoxy and free SNPs. The morphology of AgNWs separated from the mixtures was examined by TEM. Fig. 1 shows clearly the SNPs are adsorbed on the surface of AgNWs. Especially, when the amount of SNPs in the epoxy composites is increased, more SNPs are absorbed on the surface of AgNWs.

Ultrathin-section TEM was used to further study the interaction between AgNWs and SNPs in the cured hybrid epoxy/silica/AgNWs nanocomposites. Fig. 2 indicates that the SNPs are still adsorbed on the surface of AgNWs even after the curing process. The interaction between SNPs and PVP coated on AgNWs forced the SNPs to assemble at the AgNWs-epoxy interface. It is noted that the solvent evaporation and epoxy curing processes did not destroy the desired interactions between AgNWs and SNPs.

According to the attractive interactions between AgNWs and SNPs, the agglomerate-free epoxy/SNPs nanocomposites may be ideal materials to enhance the dispersion of AgNWs in epoxy composites by an easy mixing process. It is expected that the excellent dispersion and attractive interaction between silver nanowires and silica nanoparticles will lead to substantial enhancement of the thermal conductivity in these epoxy composites.

\subsection{AgNWs dispersion in epoxy composites}

SEM images exhibited in Fig. 3 give insight into the influence of different SNPs content on the dispersion state of AgNWs in epoxy composites. Without SNPs, many raw AgNWs tend to agglomerate into clusters (Fig. 3a) despite some are well-dispersed in epoxy. With 5 wt.\% SNPs, the well-dispersed AgNWs proportion increases but the AgNWs clusters remain on the 
fracture surface (Fig. 3b). However, further increasing SNPs to $10 \mathrm{wt} . \%$ and $15 \mathrm{wt} . \%$, almost homogeneous dispersions of AgNWs in epoxy matrix can be achieved (see Figs. 3c and 3d).

To further investigate the dispersion of both SNPs and AgNWs in epoxy composites, SEM and EDX analyses (Figs. 4a and 4b) were used to verify the composition of the hybrid epoxy/silica/AgNWs nanocomposites. EDX mapping images of Si and Ag are shown in Figs. $4 \mathrm{c}$ and $4 \mathrm{~d}$. The uniform distributions of $\mathrm{Ag}$ and $\mathrm{Si}$ elements on the fracture surface confirm that both AgNWs and SNPs are homogeneously dispersed in the hybrid epoxy/silica/AgNWs composites. Hence, the dispersion of AgNWs in epoxy was improved by adding SNPs which act as an efficient dispersant.

\subsection{Rheological properties of epoxy composites}

Fig. S4 shows the effects of different SNPs loading on the rheological properties of epoxy at $25{ }^{\circ} \mathrm{C}$. It is clear that high filler loading of SNPs will cause a high viscosity $\lambda$ of the hybrid epoxy/silica/AgNWs nanocomposites over the entire range of shear rates (Fig. S4a). However, once the shear rate exceeded $15.8 \mathrm{~s}^{-1}$, all epoxy composites have a low viscosity less than 20 Pa.s which is required for underfill materials.

The non-Newtonian index (n) which describes the deviation from Newtonian flow can be calculated according to the power law equation:

$$
\eta=K \gamma^{n-1}
$$

where $\eta, K$ and $\gamma$ are shear stress, consistency coefficient and shear rate, respectively. Plots of $\ln \eta$ versus $\ln \gamma$ in the pseudo-plastic flow region give a linear relationship whose slope yields $\mathrm{n}$. Generally, the smaller the $\mathrm{n}$ value, the stronger is the shear-sensitivity [30]. As shown in Fig. S4b, increasing SNPs loading decreases n, hence indicating hybrid epoxy/silica/AgNWs 
nanocomposites with more SNPs have higher shear-sensitivity. We proposed in [31] that the zero-dimension SNPs have a 'ball bearing' effect that reduces the flow resistance of AgNWs while shearing it, thus decreasing the viscosity of the hybrid epoxy/silica/AgNWs composites. As expected, the ball bearing effect of nanoparticles on viscosity becomes more obvious with increasing SNPs loading. Hence, the viscosity of the hybrids decreases more rapidly with increasing shear rate which benefits the underfill process. Furthermore, at low shear rates, the hybrids have higher viscosities than epoxy composites without or with low SNPs loading, therefore yielding better storage stability and eliminating the settlement of AgNWs.

The state of filler dispersion, aspect ratio, and interactions between filler and matrix are closely related to the rheological properties of polymer composites $[32,33]$. The rheological properties of the hybrid epoxy/silica/AgNWs composites provide another way to study the dispersion of the AgNWs in epoxy besides the SEM image analysis of the fracture surface. Generally, the lesser the frequency dependence of storage modulus G' means more restraints of the AgNWs network on the epoxy chains, indicating a better dispersion of fillers $[34,35]$. The storage moduli $\left(\mathrm{G}^{\prime}\right)$ of the hybrid epoxy/silica/AgNWs composites measured with respect to angular frequency are displayed in Fig. S5. With increasing loading of SNPs, higher G' of hybrid epoxy/silica/AgNWs nanocomposites are obtained but their frequency dependence is gradually reduced. The dynamic rheological results on the dispersion state of SNPs therefore further support the fracture surface SEM image analysis. This confirms that SNPs effectively improve the dispersion of AgNWs in epoxy matrix. Also, the AgNWs network formed in the presence of the SNPs should promote the thermal conductivity of the epoxy composites.

\subsection{Mechanical properties}


As mentioned, for the widely used high thermal conductivity but electrical insulating fillers, such as boron nitride $(\mathrm{BN})$, aluminum nitride $(\mathrm{AlN})$, aluminum oxide $\left(\mathrm{Al}_{2} \mathrm{O}_{3}\right)$ and silicon carbide ( $\mathrm{SiC}$ ), high filler loading is required to increase the thermal conductivity of epoxy composites. But high filler loading increases the viscosity of the composite and degrades its processability and mechanical properties. Besides the thermal and electrical conductivities, the effects of adding the SNPs into the hybrids on the mechanical properties, e.g., fracture toughness, fracture energy, elastic modulus and hardness, of a typical epoxy/silica/AgNWs composite with $10 \mathrm{wt} . \%$ SNPs are studied since they are critical to the mechanical reliability during service.

Fig. 5 shows both binary epoxy/AgNWs and hybrid epoxy/silica/AgNWs nanocomposites exhibit moderate improvements in fracture toughness $\left(K_{\mathrm{IC}}\right)$ and fracture energy $\left(G_{\mathrm{IC}}\right)$ with increasing AgNWs loading. At 4 vol.\% AgNWs, $K_{\text {IC }}$ of hybrid epoxy/silica/AgNWs increases from 0.821 to $0.972 \mathrm{MPa} \mathrm{m}^{1 / 2}$, and $G_{\text {IC }}$ from 235.5 to $278.3 \mathrm{~J} / \mathrm{m}^{2}$. The $K_{\text {IC }}$ results in Fig. 5 a do not show any synergistic but a near additive effect by hybridisation of zero-dimensional SNPs and one-dimensional AgNWs.

The fracture surface morphologies of binary epoxy/AgNWs and hybrid epoxy/silica/AgNWs nanocomposites are displayed in Fig. S6 which show many pulled out AgNWs and their corresponding holes. This suggests that the frictional pullout of AgNWs from epoxy is the major toughening mechanism which has increased the fracture energy $G_{\mathrm{IC}}$ $[36,37]$. In the case of SNPs absorbed onto the surfaces of AgNWs, the interface friction is increased and there are more pulled out AgNWs due to the more uniform distribution in epoxy (compared Figs. S6b to 6a). These facts increase $G_{\text {IC }}$ of hybrid epoxy/silica/AgNWs 
above the binary epoxy/AgNWs (see Fig. 5b).

The elastic modulus $\left(E_{s}\right)$ and hardness $(H)$ of the hybrid epoxy/silica/AgNWs composites were measured by nanoindentations. Fig. S7 shows higher elastic modulus as AgNWs loading is increased. For example, with 4 vol. $\%$ AgNWs, $E_{s}$ of the hybrid nanocomposite is $4.22 \mathrm{GPa}$ giving a $31.8 \%$ increase compared with neat epoxy at $3.21 \mathrm{GPa}$. The low hardness of AgNW ( $\sim 0.87 \mathrm{GPa}$ ) only leads to marginal improvement in $H$ with increasing AgNWs loading in the hybrid nanocomposites [38].

\subsection{Dynamic mechanical properties}

The storage modulus $\left(E^{\prime}\right)$ and loss tangent $(\tan \delta)$ as a function of temperature for the F100/AgNWs composites (F100 is epoxy/SNPs (10 wt.\%)) with different loading of AgNWs are shown in Fig. 6. Adding higher modulus AgNWs to the hybrid epoxy/silica/AgNWs composites exhibit continuous improvements in $E^{\prime}$ in the glass region. For example, at $40{ }^{\circ} \mathrm{C}$, $E^{\prime}$ increases from $2.85 \mathrm{GPa}$ for neat epoxy to $3.74 \mathrm{GPa}$ for the hybrid with 4 vol. $\%$ AgNWs. Such improvement is caused by the uniformly dispersed AgNWs and enhanced interfacial adhesion for load transfer from the matrix to the AgNWs. Also, $T_{\mathrm{g}}$ increases from $127.2{ }^{\circ} \mathrm{C}$ to $135.3{ }^{\circ} \mathrm{C}$ when adding 2 vol.\% AgNWs to the hybrid epoxy/silica/AgNWs nanocomposite, which is ascribed to the segmental confinement of the well-dispersed AgNWs. Note that $T_{\mathrm{g}}$ shows a large reduction once AgNWs are increased to 4 vol.\% because of the increased free volume and decreased crosslinking degree of epoxy matrix effected by the viscosity increase due to further addition of AgNWs. However, the $T_{\mathrm{g}}$ values for all hybrid epoxy/silica/AgNWs composites with different amount of AgNWs are higher than $125{ }^{\circ} \mathrm{C}$ which is required for underfill materials. 


\subsection{Thermal conductivity $(\kappa)$ and electrical resistivity $(\rho)$ of epoxy composites}

The thermal conductivities of neat epoxy and SNP-modified epoxy are plotted as a function of AgNWs loading in Fig. 7a. Clearly, for a given amount of SNPs in epoxy, the thermal conductivity $\kappa$ increases with increasing AgNWs. Similarly, for a given AgNWs loading, the thermal conductivity of the hybrids increases with increasing SNPs loading. It is found that the epoxy/AgNWs composite has $\kappa=0.705 \mathrm{~W} / \mathrm{mK}$ with 4 vol. $\%$ AgNWs. However, adding 15 wt.\% SNPs to this binary composite gives a higher $\kappa=1.081 \mathrm{~W} / \mathrm{mK}$ for the hybrid epoxy/silica/AgNWs composite that has satisfied the requirement $\kappa>1.0 \mathrm{~W} / \mathrm{mK}$ for underfill materials (Fig. 7a). It is also noted that the hybrid epoxy/silica/AgNWs composites with 15 wt.\% and 10 wt.\% SNPs at 2 vol.\% AgNWs possess higher thermal conductivities than epoxy/AgNWs with 4 vol.\% AgNWs. These results confirm that reducing the AgNWs loading will not deteriorate the thermal conductivity which can be compensated by adding more SNPs. The thermal conductivity enhancement factor (see Fig. $7 b)$ defined as $\left[\left(\kappa_{\text {hybrid }}-\kappa_{\text {binary }}\right) /\right.$ $\kappa_{\text {binary }}$ or $\left.\left(\kappa_{\text {binary }}-\kappa_{\text {epoxy }}\right) / \kappa_{\text {epoxy }}\right]$ where $\kappa_{\text {binary }}$ and $\kappa_{\text {epoxy }}$ are given in Fig. S8 and the first block of bar charts (0 wt.\% AgNWs) in Fig. 7a. It is evident that the enhancements of the hybrid epoxy/silica/AgNWs nanocomposites with the same AgNWs loading are larger with increasing SNPs, especially at $10 \mathrm{wt} . \%$ and $15 \mathrm{wt} . \%$. However, it should be cautioned that with too many SNPs, e.g., 40 wt.\% SNPs in the hybrid epoxy/silica/AgNWs composites having 4 vol.\% AgNWs, the thermal conductivity enhancement factor is lower than the same hybrid with 10 wt.\% SNPs due to the much higher viscosity forming voids inside the hybrid with 40 wt.\% SNPs.

Fig. S9 shows that addition of SNPs in epoxy improves the Young's modulus from 2.65 
(neat epoxy) to $4.33 \mathrm{GPa}(40 \mathrm{wt} . \%)$. The enhanced modulus of epoxy matrix is beneficial to alleviate the modulus mismatch between neat epoxy ( $3 \mathrm{GPa})$ and AgNWs ( 102 GPa). Previous work [39-41] has shown that reducing the elastic modulus mismatch is equivalent to decreasing the acoustic impedance mismatch between filler and matrix such that the phonon vibrational spectrum is shifted towards higher frequencies, yielding a better spectral overlap between high modulus AgNWs and low modulus epoxy matrix and decreasing the interfacial thermal resistance. Moreover, the SNPs assembled at the AgNWs/epoxy matrix interface which have a modulus of $70 \mathrm{GPa}$ also act like a springboard to reduce the phonon propagation barrier and increase the overlap of the phonon vibrational spectra in the interface regions [23], thus decreasing the interfacial thermal resistance and improving the thermal conductivity of the hybrid epoxy/silica/AgNWs nanocomposites.

Fig. S10 shows the volume electrical resistivity $(\rho)$ of neat epoxy and hybrid epoxy/silica/AgNWs nanocomposites containing various loading of AgNWs. When AgNWs is less than 4 vol.\%, adding SNPs to epoxy matrix has little influence on $\rho$ of epoxy/AgNWs and hybrid epoxy/silica/AgNWs composites. However, with 4 vol.\% AgNWs, $\rho$ of epoxy/AgNWs and hybrid epoxy/silica/AgNWs (5 wt.\% SNPs) decrease by almost eight and seven orders of magnitude, from $1.15 \times 10^{12} \Omega \mathrm{cm}$ to $6.58 \times 10^{3} \Omega \mathrm{cm}$ and $1.84 \times 10^{4} \Omega \mathrm{cm}$, respectively. These results can be attributed to the formation of electrical networks when the AgNWs loading has surpassed the electrical percolation threshold. It is particularly interesting to note that when the SNPs are increased to $10 \mathrm{wt} . \%$ and $15 \mathrm{wt} . \%$, the electrical resistivity $\rho$ of the hybrid epoxy/silica/AgNWs composites are $9.33 \times 10^{11} \Omega . \mathrm{cm}$ and $2.62 \times 10^{12} \Omega . \mathrm{cm}$, indicating they are still electrical insulating and retain the electrical insulation of the epoxy 
matrix. This suggests that above $10 \mathrm{wt} \%$ the SNPs can assemble at the AgNWs/epoxy interface acting as an electrical insulation layer effectively interrupting the electron flow between adjacent AgNWs to disrupt the formation of electrical networks.

\section{Concluding remarks}

In this study, we have demonstrated a facile and effective strategy for improving the dispersion of AgNWs through blending with SNPs-modified epoxy matrix to fabricate isotropic thermally conductive epoxy/AgNWs composites. The interaction between the SNPs and polyvinyl pyrrolidone (PVP) which is coated on AgNWs facilitate the SNPs to assemble at the AgNWs/epoxy interface, serving an important role in avoiding the agglomeration of AgNWs and the formation of an AgNWs electrical conductive network. Compared with the binary epoxy/AgNWs composites, the hybrid epoxy/silica/AgNWs composites exhibit clear advantages in thermal conductivity, fracture toughness and fracture energy. Furthermore, with increasing AgNWs loading, the storage modulus, elastic modulus and hardness of the hybrid epoxy/silica/AgNWs composites are gradually increased without degrading the mechanical properties of epoxy composites. Hence, the results of this work provide a simple and effective method to fabricate high-performance epoxy-based underfill materials with excellent thermal conductivity, high electrical insulation, superior fracture toughness/fracture energy and good rheological properties for use by the IC electronic packaging industries (see Fig. 8).

\section{Acknowledgments}

We are grateful to the Major International (Regional) Joint Research Project of the National 
Science Foundation of China (Grant Nos. 51210004) for the support of this work. We also acknowledge access to SEM, TEM and EDAX facilities of the Analytical and Testing Center of Huazhong University of Science and Technology. CC was an Occupational Trainee when part of this work was conducted in the CAMT at the University of Sydney.

\section{References}

[1] Moore AL, Shi L. Emerging challenges and materials for thermal management of electronics. Mater Today 2014;17(4):163-74.

[2] Shahil KM, Balandin AA. Graphene-multilayer graphene nanocomposites as highly efficient thermal interface materials. Nano Lett 2012;12(2):861-7.

[3] Xu J, Munari A, Dalton E, Mathewson A, Razeeb KM. Silver nanowire array-polymer composite as thermal interface material. J Appl Phys 2009;106(12):124310.

[4] Lin Z, McNamara A, Liu Y, Moon K-s, Wong C-P. Exfoliated hexagonal boron nitridebased polymer nanocomposite with enhanced thermal conductivity for electronic encapsulation. Compos Sci Technol 2014; 90:123-8.

[5] Ahmad FN, Jaafar M, Palaniandy S, Azizli KAM. Effect of particle shape of silica mineral on the properties of epoxy composites. Compos Sci Technol 2008;68(2):346-53.

[6] Xu Y, Chung DDL, Mroz C. Thermally conducting aluminum nitride polymer-matrix composites. Composites A 2001;32(12):1749-57.

[7] Yu JH, Huang XY, Wang LC, Peng P, Wu C, Jiang PK, et al. Preparation of hyperbranched aromatic polyamide grafted nanoparticles for thermal properties reinforcement of epoxy composites. Polym Chem 2011;2(6):1380-8.

[8] Yang K, Gu MY. Enhanced thermal conductivity of epoxy nanocomposites filled with hybrid filler system of triethylenetetramine-functionalized multi-walled carbon nanotube/ silane-modified nano-sized silicon carbide. Composites A 2010; 41 (2):215-21.

[9] Cao J-P, Zhao J, Zhao XD, You F, Yu H, Hu G-H, et al. High thermal conductivity and high electrical resistivity of poly(vinylidene fluoride)/polystyrene blends by controlling 
the localization of hybrid fillers. Compos Sci Technol 2013;89:142-8.

[10] Cui W, Du F, Zhao J, Zhang W, Yang Y, Xie X, Mai Y-W. Improving thermal conductivity while retaining high electrical resistivity of epoxy composites by incorporating silica-coated multi-walled carbon nanotubes. Carbon 2011;49(2):495-500.

[11] Xie XL, Mai Y-W, Zhou XP. Dispersion and alignment of carbon nanotubes in polymer matrix: A review. Mat Sci Eng R 2005;49(4):89-112.

[12] Zhao JC, Du FP, Zhou XP, Cui W, Xie XL, Mai Y-W, et al. Thermal conductive and electrical properties of polyurethane/hyperbranched poly(urea-urethane)-grafted multiwalled carbon nanotube composites. Composites B 2011;42(8):2111-6.

[13] Han Z, Fina A. Thermal conductivity of carbon nanotubes and their polymer nanocomposites: A review. Prog Polym Sci 2011;36(7):914-44.

[14] Zhong H, Lukes JR. Interfacial thermal resistance between carbon nanotubes: Molecular dynamics simulations and analytical thermal modeling. Phys Rev B 2006; 74(12): 125403.

[15] Li Q, Liu C, Fan S. Thermal boundary resistances of carbon nanotubes in contact with metals and polymers. Nano Lett 2009;9(11):3805-9.

[16] Balandin AA. Thermal properties of graphene and nanostructured carbon materials. Nat Mater 2011;10(8):569-81.

[17] Zhi C, Bando Y, Terao T, Tang C, Kuwahara H, Golberg D. Towards thermoconductive, electrically insulating polymeric composites with boron nitride nanotubes as fillers. Adv Funct Mater 2009;19(12):1857-62.

[18] Shtein M, Nadiv R, Buzaglo M, Kahil K, Regev O. Thermally conductive graphenepolymer composites: Size, percolation, and synergy effects. Chem Mater 2015;27(6):2100-6.

[19] Li Q, Guo Y, Li W, Qiu S, Zhu C, Wei X, et al. Ultrahigh thermal conductivity of assembled aligned multilayer graphene/epoxy composite. Chem Mater 2014; 26(15):4459-65.

[20] Nam S, Cho HW, Lim S, Kim D, Kim H, Sung BJ. Enhancement of electrical and thermomechanical properties of silver nanowire composites by the introduction of nonconductive nanoparticles: experiment and simulation. ACS nano 2013;7(1):851-6. 
[21] Shi H-Y, Hu B, Yu X-C, Zhao R-L, Ren X-F, Liu S-L, et al. Ordering of disordered nanowires: Spontaneous formation of highly aligned, ultralong ag nanowire films at oilwater-air interface. Adv Funct Mater 2010;20(6):958-64.

[22] Zhao JC, Du FP, Cui W, Zhu P, Zhou XP, Xie XL. Effect of silica coating thickness on the thermal conductivity of polyurethane/ $\mathrm{SiO}_{2}$ coated multiwalled carbon nanotube composites. Composites A 2014;58:1-6.

[23] Chen C, Tang Y, Ye YS, Xue Z, Xue Y, Xie X, Mai Y-W. High-performance epoxy/silica coated silver nanowire composites as underfill material for electronic packaging. Compos Sci Technol 2014;105:80-5.

[24] Kinloch AJ, Lee JH, Taylor AC, Egan D, Eger C, Sprenger S. Nanoadhesives: toughness and high strength. Adhaes Kleb Dicht 2003;47(9):20-4.

[25] Yin YD, Lu Y, SunYG, Xia YN. Silver nanowires can be directly coated with amorphous silica to generate well-controlled coaxial nanocables of silver/silica. Nano Lett 2002;2(4):427-30.

[26] Sun YX, Mayers B, Herricks T, Xia YN. Polyol Synthesis of uniform silver nanowires: A plausible growth mechanism and the supporting evidence. Nano Lett 2003;3(7):955-960.

[27] Sun YX, Xia YN. Large-scale synthesis of uniform silver nanowires through a soft, self-seeding, polyol process. Adv Mater 1991;14(11):833-7.

[28] Barnett K G, Cosgrove T, Vincent B, et al. The measurement of the polymer-bound fraction at the solid-liquid interface by pulsed nuclear magnetic resonance. Macromolecules 1981; 14(4): 1018-20.

[29] Zhang Q, Zhang T, Ge J, et al. Permeable silica shell through surface-protected etching. Nano Lett 2008; 8(9): 2867-71.

[30] Huang LP, Zhou XP, Cui W, Xie XL, Tong SY. Maleic anhydride-grafted linear lowdensity polyethylene with low gel content. Polym Eng Sci 2009;49(4):673-9.

[31] Xie XL, Liu QX, Li RKY, Zhou XP, Zhang QX, Yu ZZ, et al. Rheological and mechanical properties of $\mathrm{PVC} / \mathrm{CaCO}_{3}$ nanocomposites prepared by in situ polymerization. Polymer 2004;45(19):6665-73.

[32] Moniruzzaman M, Winey KI. Polymer nanocomposites containing carbon nanotubes. 
Macromolecules 2006;39(16):5194-205.

[33] Kim H, Macosko CW. Morphology and properties of polyester/exfoliated graphite nano -composites. Macromolecules 2008;41(9):3317-27.

[34] Wagener R, Reisinger TJG. A rheological method to compare the degree of exfoliation of nanocomposites. Polymer 2003;44(24):7513-8.

[35] Du F, Scogna RC, Zhou W, Brand S, Fischer JE, Winey KI. Nanotube networks in polymer nanocomposites: Rheology and electrical conductivity. Macromolecules 2004; 37(24):9048-55

[36] Wetzel B, Haupert F, Qiu Zhang M. Epoxy nanocomposites with high mechanical and tribological performance. Compos Sci Technol 2003;63(14):2055-67.

[37] Sun L, Gibson RF, Gordaninejad F, Suhr J. Energy absorption capability of nanocomposites: A review. Compos Sci Technol 2009;69(14):2392-409.

[38] Li X, Gao H, Murphy CJ, Caswell KK. Nanoindentation of silver nanowires. Nano lett 2003;3(11):1495-8.

[39] Peters JE, Papavassiliou DV, Grady BP. Unique thermal conductivity behavior of single-walled carbon nanotube-polystyrene composites. Macromolecules 2008;41(20): 7274-7.

[40] Cahill DG, Ford WK, Goodson KE, Mahan GD, Majumdar A, Maris HJ, et al. Nanoscale thermal transport. J Appl Phys 2003;93(2):793-818.

[41] $\mathrm{Hu}$ M, Shenogin S, Keblinski P. Molecular dynamics simulation of interfacial thermal conductance between silicon and amorphous polyethylene. Appl Phys Lett 2007;91(24): 241910.

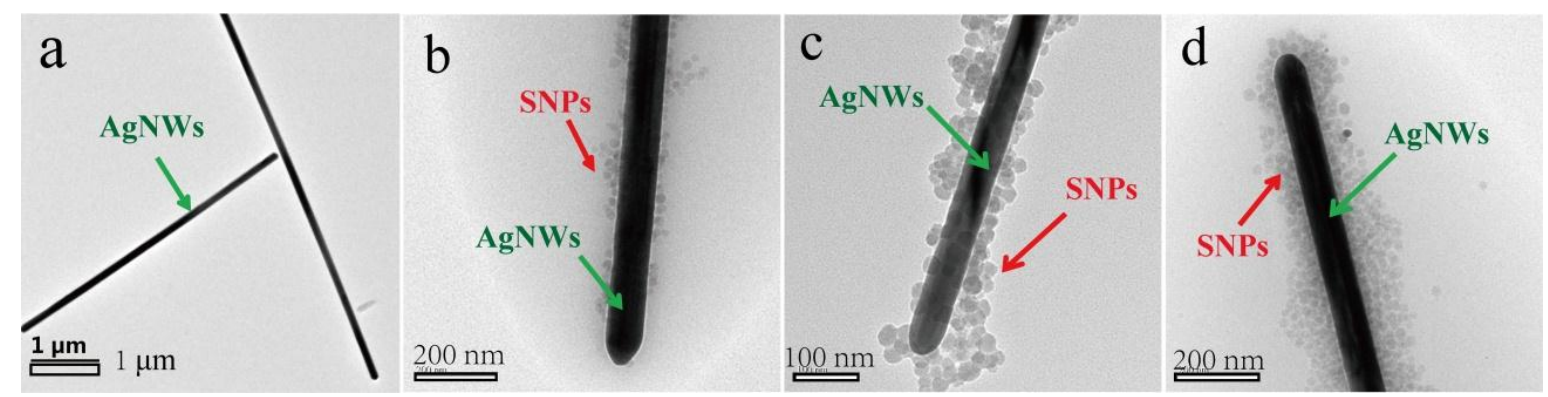

Fig. 1 TEM images of (a) AgNWs and (b-d) AgNWs separated from the mixtures after being blended with F050, F100 and F150. 

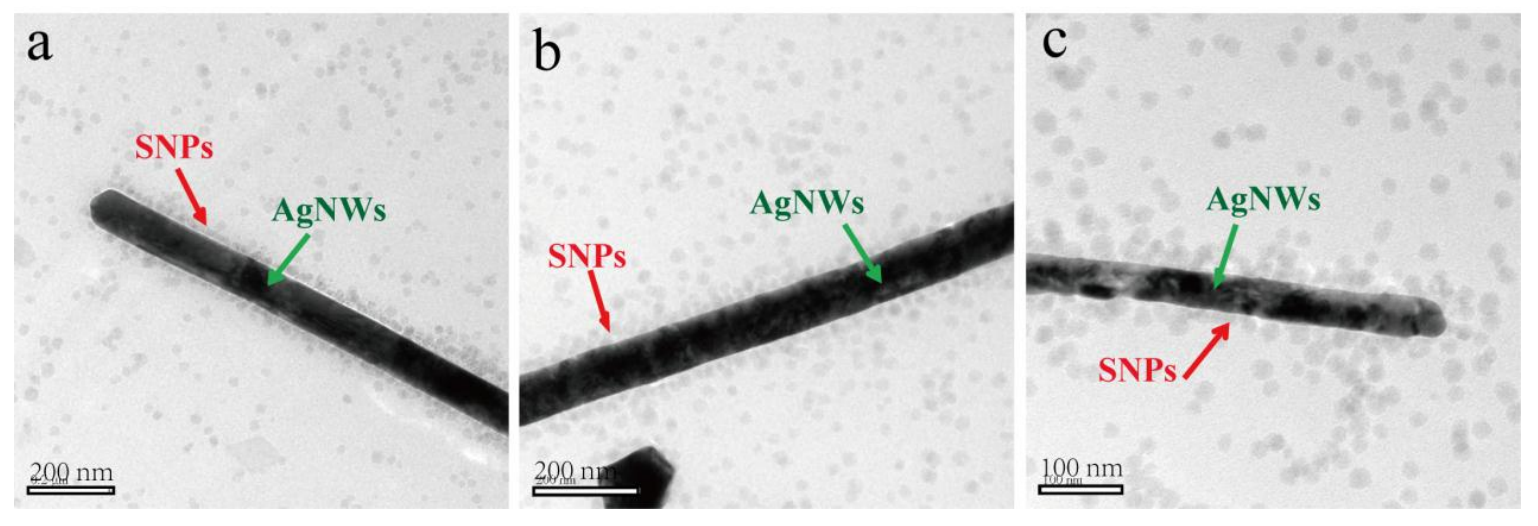

Fig. 2 Ultrathin section TEM images of cured epoxy/silica/AgNWs nanocomposites with (a) 5, (b) 10 and (c) $15 \mathrm{wt} . \%$ of SNPs at a fixed AgNWs loading of 4 vol.\%.
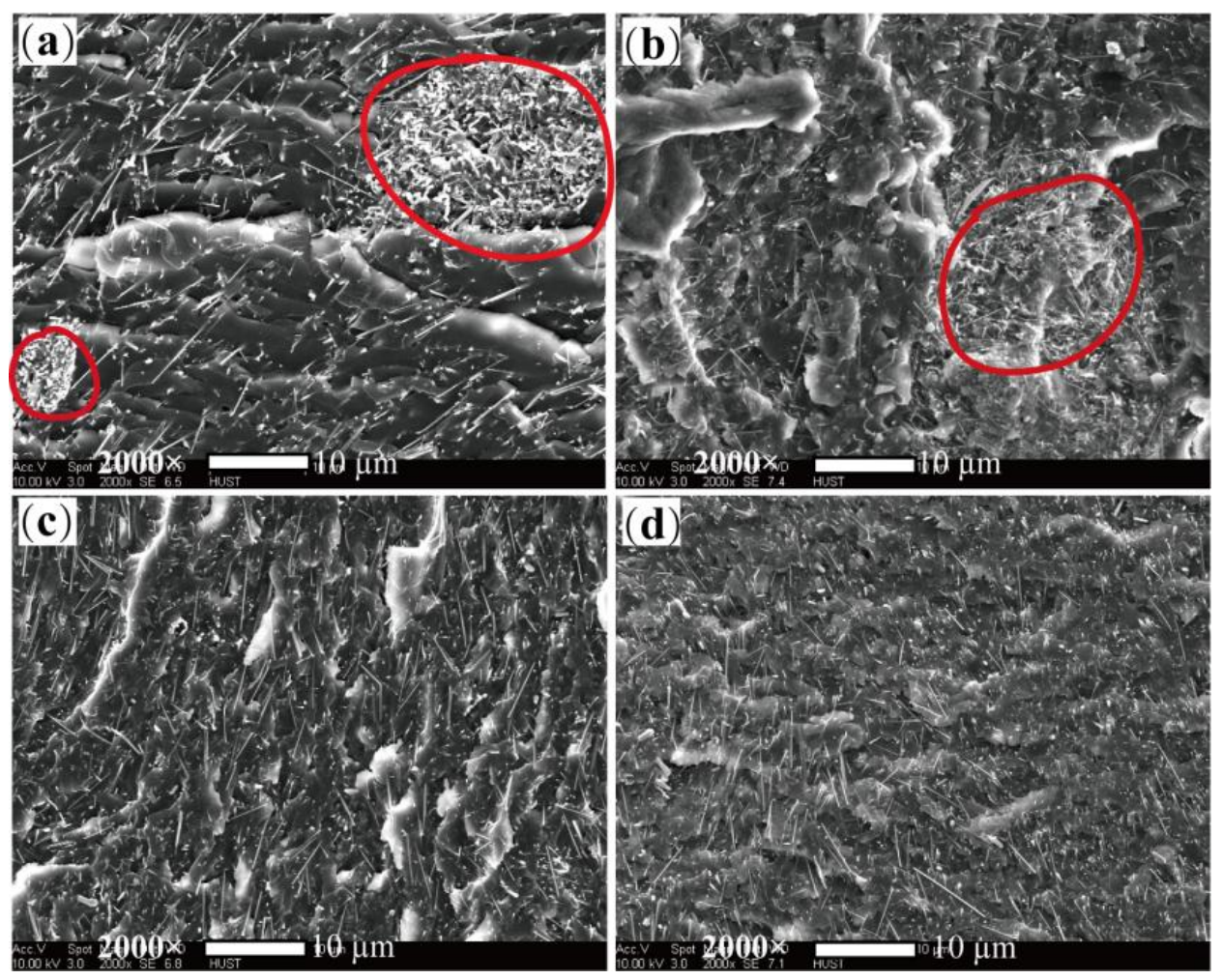

Fig. 3 SEM images for fracture surfaces of cured hybrid epoxy/silica/AgNWs nanocomposites with (a) 0, (b) 5, (c) 10 and (d) 15 wt.\% of SNPs at fixed AgNWs loading of 4 vol.\%. Scale bar is $10 \mu \mathrm{m}$ for each case. 

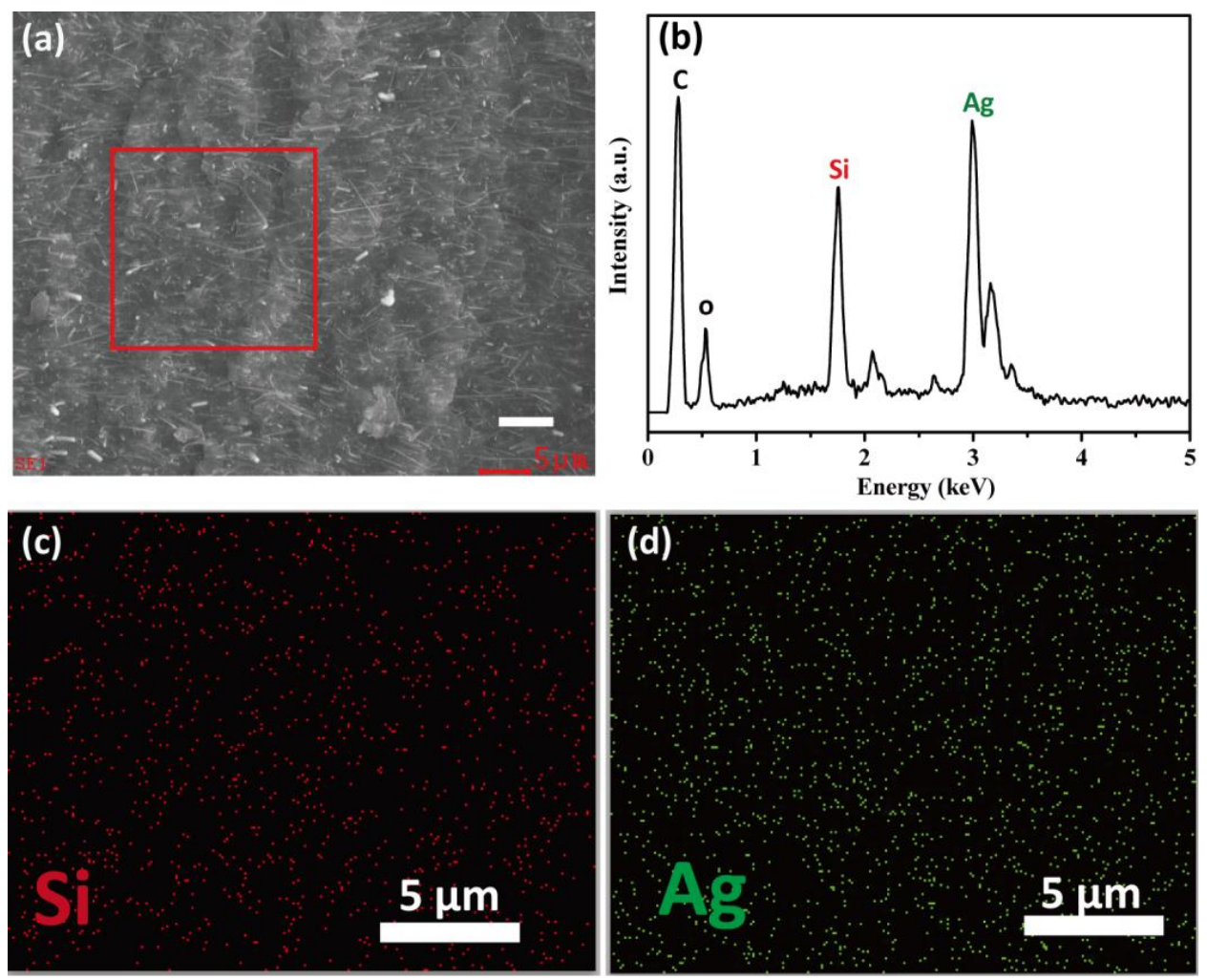

Fig. 4 (a) SEM image, (b) EDAX spectrum of selected red square highlighted area, and (c-d) EDX mapping images of $\mathrm{Si}$ and $\mathrm{Ag}$ elements on fracture surface of cured hybrid epoxy/silica/AgNWs nanocomposite with 10 wt.\% SNPs and 4 vol.\% AgNWs.
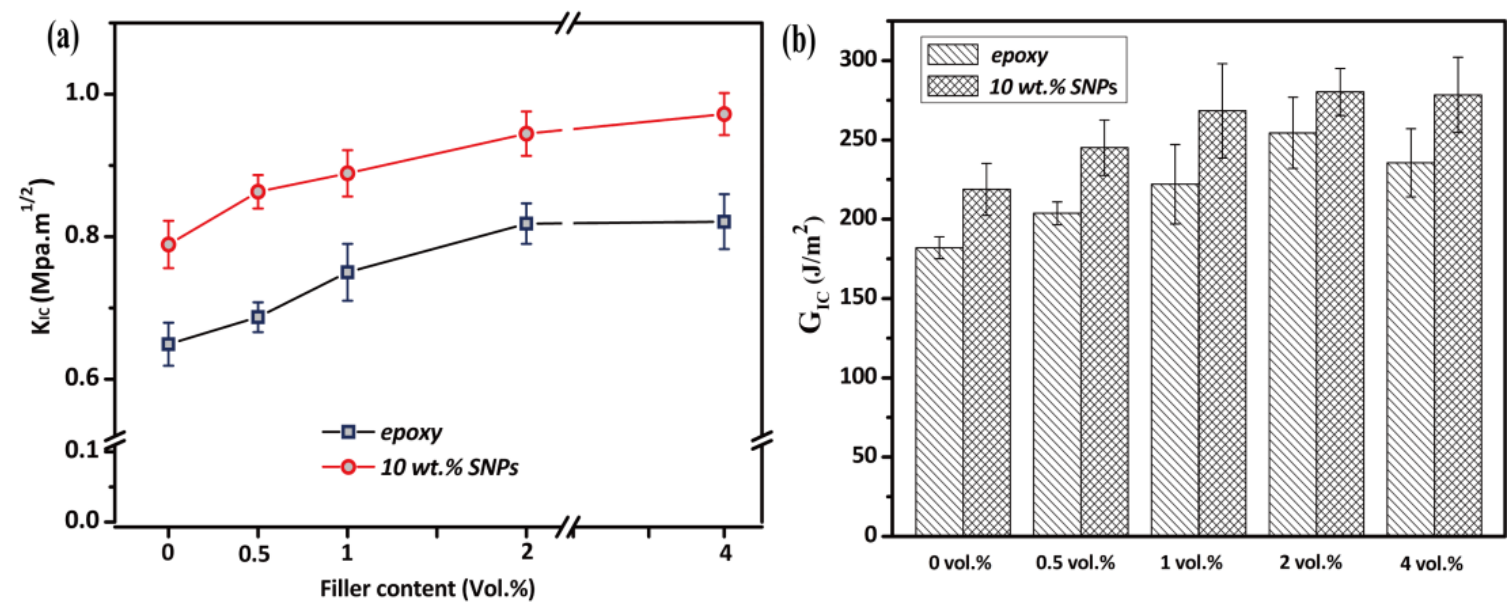

Fig. 5 (a) Fracture toughness and (b) fracture energy of epoxy/AgNWs nanocomposites and hybrid epoxy/silica/AgNWs nanocomposites with $10 \mathrm{wt}$ \% SNPs as a function of AgNWs content. 

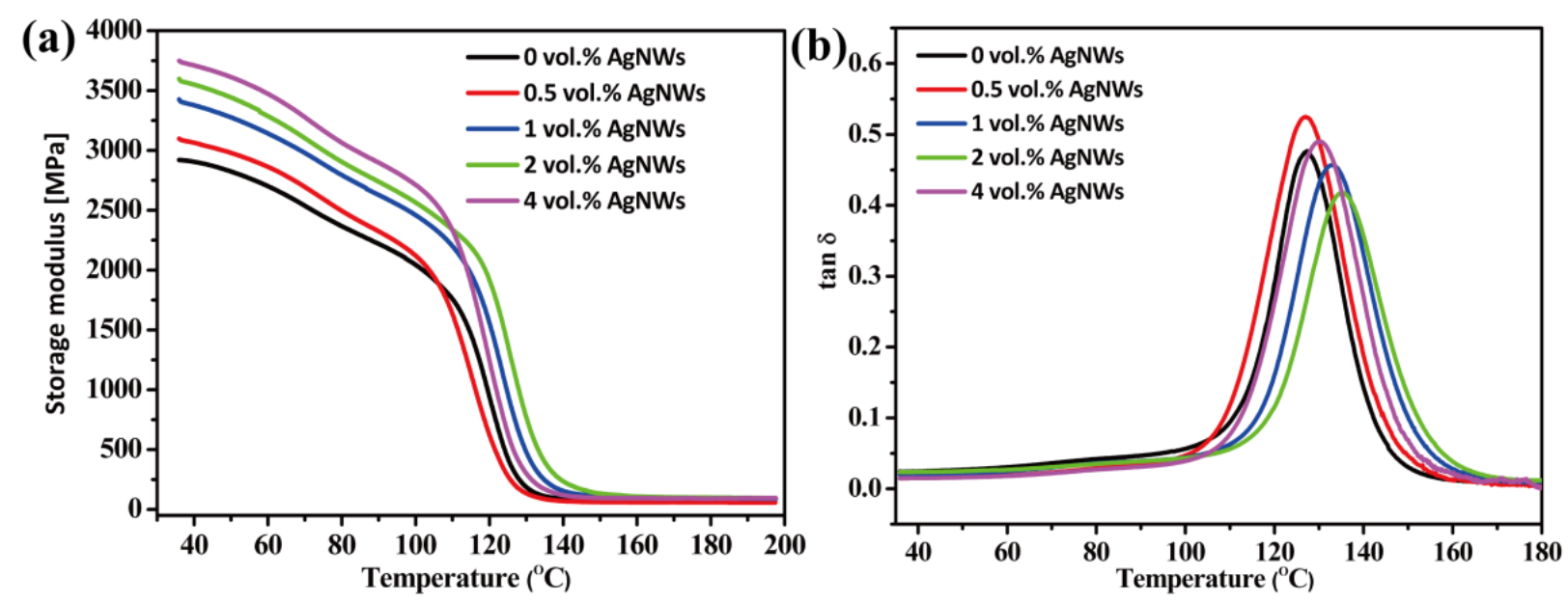

Fig. 6 (a) Storage modulus and (b) tan $\delta$ versus temperature for hybrid epoxy/silica/AgNWs nanocomposites with $10 \mathrm{wt} . \%$ SNPs as a function of AgNWs contents.
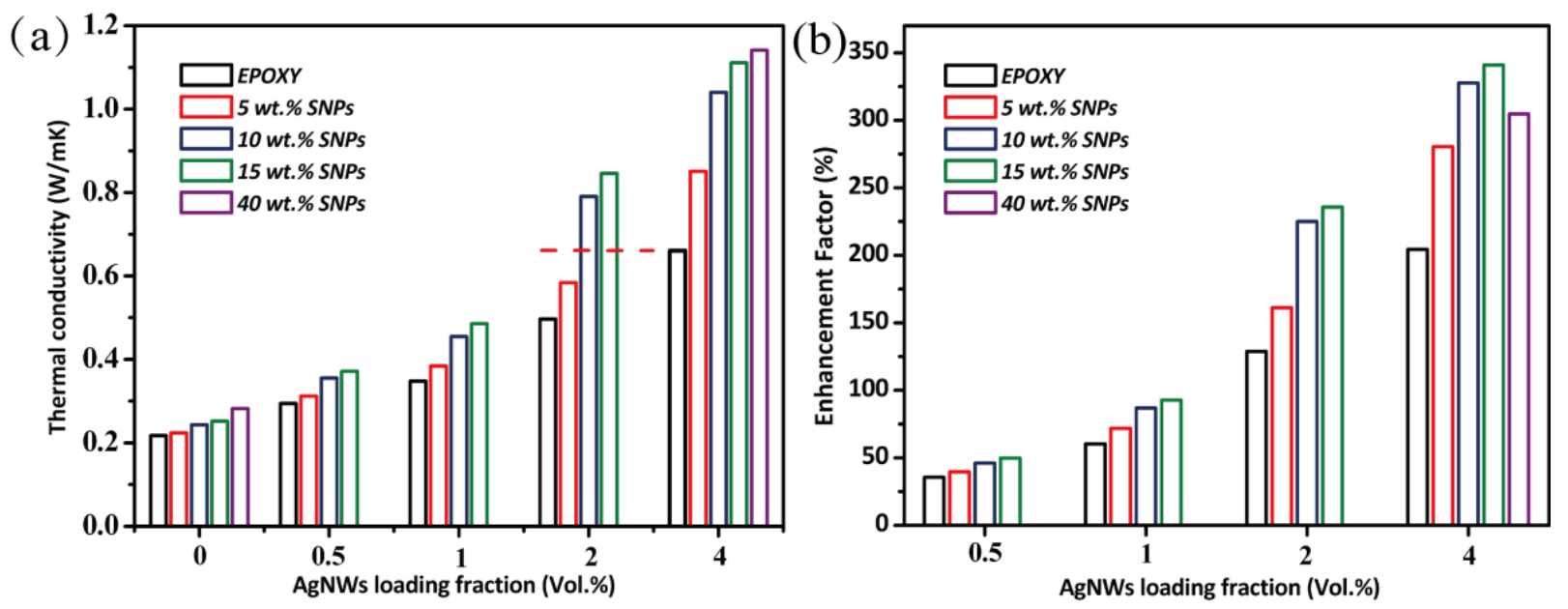

Fig. 7 (a) Thermal conductivity and (b) thermal conductivity enhancement of hybrid epoxy/silica/AgNWs nanocomposites filled with 0.5, 1, 2 and 4 vol.\% of AgNWs compared to neat epoxy. 

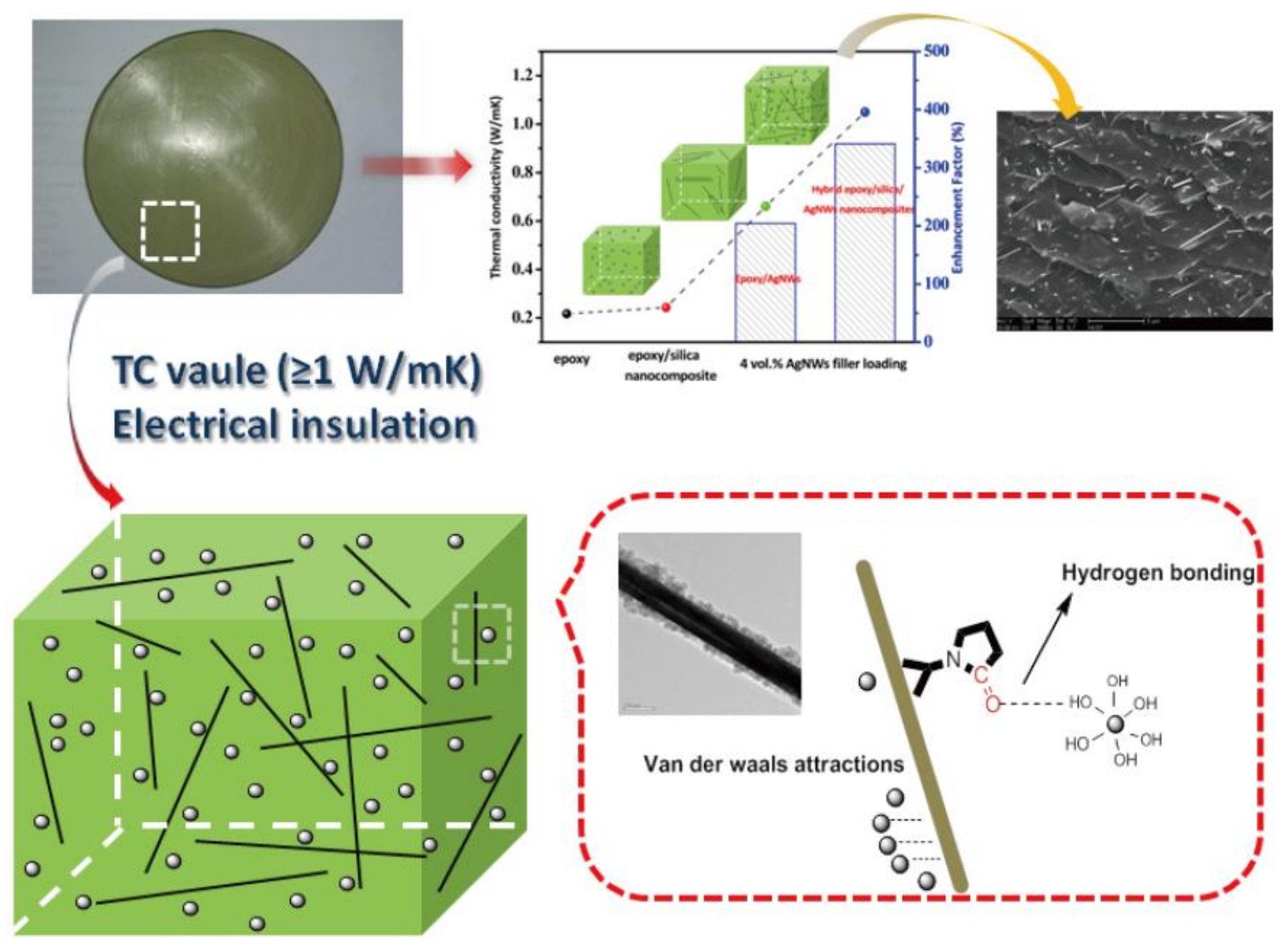

Fig. 8 Design of high thermal conductivity but electrical insulating epoxy-based underfill electronic packaging materials. 


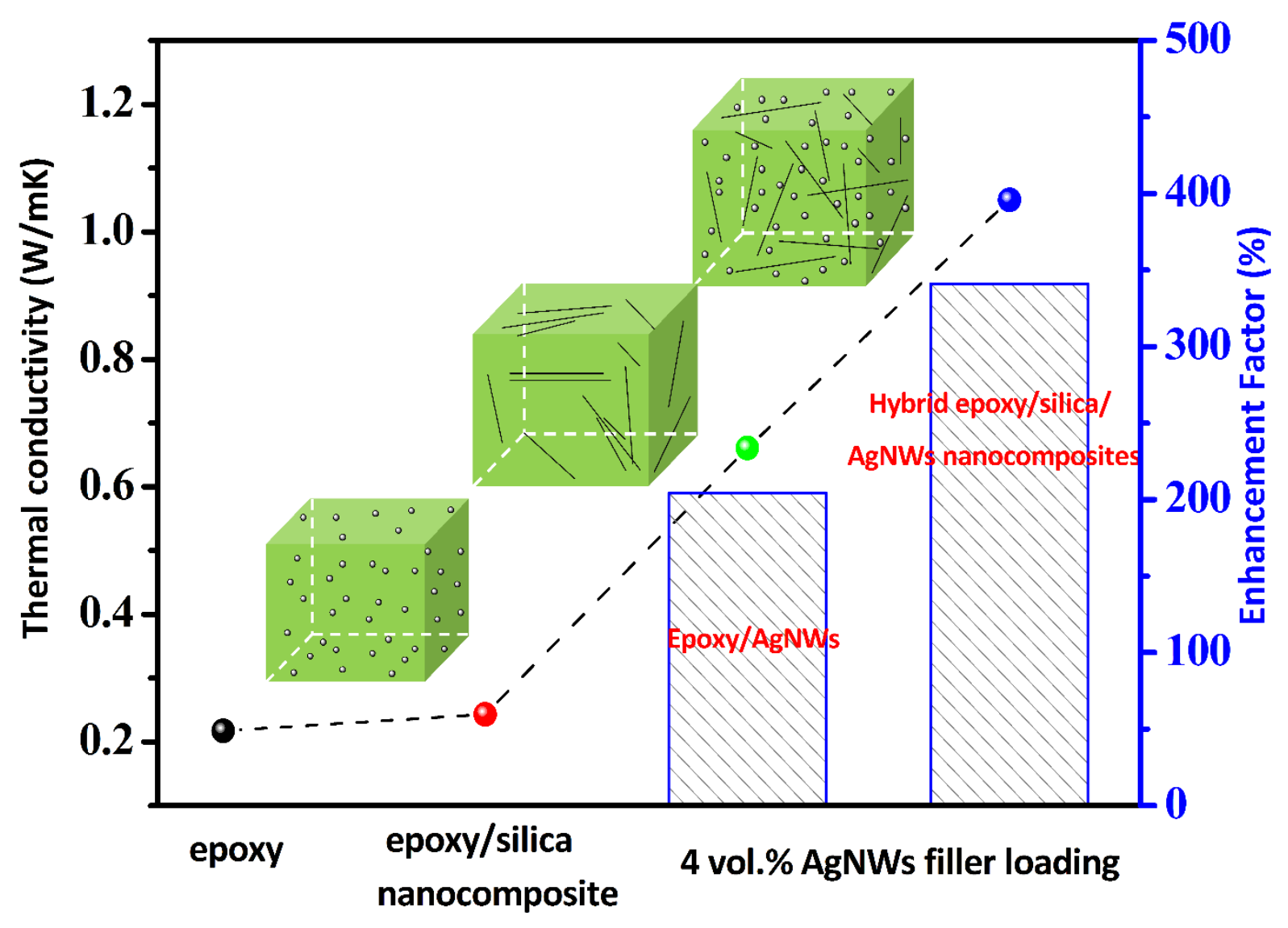

